Real Analysis Exchange

Vol. 26(1), 2000/2001, pp. 391-400

Lech Bartłomiejczyk, Institute of Mathematics, Silesian University, Bankowa 14, PL-40-007 Katowice, Poland. e-mail: lech@gate.math.us.edu.pl

\title{
ITERATIVE ROOTS WITH BIG GRAPH
}

\begin{abstract}
Let $g: X \rightarrow X$ be a bijection and $n \geq 2$ be a fixed integer. We consider the equation of iterative roots $\varphi^{n}(x)=g(x)$ and we look for its solution with big graph: big from the point of view both of topology and measure theory.
\end{abstract}

\section{Introduction}

Let $X$ and $Y$ be two sets and $\mathcal{R}$ be a family of subsets of $X \times Y$. We say that $\varphi: X \rightarrow Y$ has a big graph with respect to $\mathcal{R}$ if the graph $\operatorname{Gr} \varphi$ of $\varphi$ meets every set of $\mathcal{R}$. We are interested in finding conditions under which the functional equation of iterative roots

$$
\varphi^{n}(x)=g(x)
$$

has a solution with big graph with respect to a sufficiently large family. Well known results on additive functions with big graph are due to F. B. Jones [6] (see also [12]). Solutions with big graph for some iterative functional equation were obtained in [8], [16], [1]-[4].

\section{Main Result}

Let $X$ be a nonempty set, $g: X \rightarrow X$ be a given bijection (one-to-one and onto) and $n \geq 2$ be an integer. We start with recalling the well known theorem of S. Łojasiewicz ([15], [11], [19]) concerning the iterative roots. To formulate it, for every positive integer $k$ let $L_{k}$ denote the (cardinal) number of $k$-cycles of $g$ and $L_{0}$ denote the number of infinite orbits of $g$. Note that any infinite

\footnotetext{
Key Words: iterative root, big graph.

Mathematical Reviews subject classification: Primary 39B12, 39B22, 39B52; Secondary 26A30

Received by the editors May 4, 1999

*This research was supported by the Silesian University Mathematics Institute in the Iterative Functional Equations program.
} 
orbit of $g$ is simply the sequence of the form $\left(g^{k}(x): k \in \mathbb{Z}\right)$ and every finite orbit of $g$ is a cycle. Put $d_{0}=n$ and $d_{k}=\frac{n}{n_{k}}$ for $k \in \mathbb{N} \backslash\{0\}$, where $n_{k}$ is the largest divisor of $n$ relative prime to $k$.

Theorem (S. Łojasiewicz). Equation (1) has a solution $\varphi: X \rightarrow X$ iff for every nonnegative integer $k$ either $L_{k}$ is infinite or $L_{k}$ is divisible by $d_{k}$.

We are interested in finding special solutions of equation (1) and it is obvious that our assumptions must be stronger than Lojasiewicz ones: they read as follows.

$\left(\mathrm{H}_{1}\right)$ The set $X$ is uncountable and $g: X \rightarrow X$ is a bijection.

$\left(\mathrm{H}_{2}\right)$ There exists a $k_{0} \in \mathbb{N}$ such that

$$
\begin{aligned}
\operatorname{card} X & =L_{k_{0}}, \\
\sum_{k \neq k_{0}} L_{k} & <\operatorname{card} X
\end{aligned}
$$

and for $k \neq k_{0}$ either $L_{k}$ is infinite or $L_{k}$ is divisible by $d_{k}$.

For any set $R \subset X \times X$ and $y \in X, R^{y}$ denotes horizontal section of $R$, i.e., the set $\{x \in X:(x, y) \in R\}$. The following is the main result of this paper.

Theorem 1. Assume $\left(\mathrm{H}_{1}\right),\left(\mathrm{H}_{2}\right)$ and let $\mathcal{R}$ be a family of subsets of $X \times X$ such that

$$
\operatorname{card} \mathcal{R} \leq \operatorname{card} X
$$

and

$$
\operatorname{card}\left\{y \in X: \operatorname{card} R^{y}=\operatorname{card} X\right\}=\operatorname{card} X \text { for } R \in \mathcal{R} .
$$

Then there exists a solution $\varphi: X \rightarrow X$ of (1) which has a big graph with respect to $\mathcal{R}$.

Proof. We start with some notations. The set of all periodic points of $g$ with period $p$ will be denoted by $\operatorname{Per}(g, p)$, i.e.,

$$
\operatorname{Per}(g, p)=\left\{x \in X: g^{p}(x)=x, g^{k}(x) \neq x \text { for } k=1, \ldots, p-1\right\} ;
$$

moreover we put $\operatorname{Per} g=\bigcup_{p=1}^{\infty} \operatorname{Per}(g, p)$. Let

$$
A= \begin{cases}\operatorname{Per}\left(g, k_{0}\right), & \text { if } k_{0} \neq 0, \\ X \backslash \operatorname{Per} g, & \text { if } k_{0}=0,\end{cases}
$$


where $k_{0} \in \mathbb{N}$ satisfies the requirements stated in $\left(\mathrm{H}_{2}\right)$. According to $(2)$ and $\left(\mathrm{H}_{1}\right)$ we have

$$
\operatorname{card} A=\left\{\begin{array}{ll}
k_{0} \cdot L_{k_{0}}, & \text { if } k_{0} \neq 0, \\
\aleph_{0} \cdot L_{0}, & \text { if } k_{0}=0,
\end{array}=L_{k_{0}}=\operatorname{card} X\right.
$$

Hence and from (3) for $A_{-1}=X \backslash A$ we get

$$
\operatorname{card} A_{-1}<\operatorname{card} X
$$

Applying Eojasiewicz's theorem to the function $\left.g\right|_{A_{-1}}$ we obtain a function $\varphi_{-1}: A_{-1} \rightarrow A_{-1}$ such that $\varphi_{-1}^{n}=g \mid A_{-1}$.

Let $\gamma$ be the smallest ordinal such that its cardinal $\bar{\gamma}$ equals that of $\mathcal{R}$ and let $\left(R_{\alpha}: \alpha<\gamma\right)$ be a one-to-one transfinite sequence of all the elements of $\mathcal{R}$. Similarly, let $\delta$ be the smallest ordinal with $\bar{\delta}=\operatorname{card} A$ and $\left(x_{\alpha}: \alpha<\delta\right)$ be a one-to-one sequence of all the elements of $A$. According to (4) and (6), $\gamma \leq \delta$. Define now a sequence $\left(A_{\alpha}: \alpha<\delta\right)$ of countable subsets of $A$ and a sequence $\left(\varphi_{\alpha}: \alpha<\delta\right)$ of functions $\varphi_{\alpha}: A_{\alpha} \rightarrow A_{\alpha}$ such that for every $\alpha<\delta$ the following conditions (8)-(11) hold:

$$
\begin{aligned}
g\left(A_{\alpha}\right) & =A_{\alpha}, \\
\varphi_{\alpha}^{n} & =\left.g\right|_{A_{\alpha}}, \\
A_{\beta} \cap A_{\alpha} & =\emptyset \text { for } \beta<\alpha, \\
\left\{x_{\beta}: \beta \leq \alpha\right\} & \subset \bigcup_{\beta \leq \alpha} A_{\beta},
\end{aligned}
$$

and, for every $\alpha<\gamma$,

$$
\operatorname{Gr} \varphi_{\alpha} \cap R_{\alpha} \neq \emptyset
$$

Suppose $\alpha<\delta$ and that we have already defined suitable $A_{\beta}$ 's and $\varphi_{\beta}$ 's for every $\beta<\alpha$. According to $\left(\mathrm{H}_{1}\right)$ we have

$$
\operatorname{card}\left(\bigcup_{\beta<\alpha} A_{\beta}\right) \leq \bar{\alpha} \cdot \aleph_{0}=\max \left\{\aleph_{0}, \bar{\alpha}\right\}<\bar{\delta}=\operatorname{card} A
$$

Let $\eta<\delta$ be the first ordinal such that $x_{\eta} \notin \bigcup_{\beta<\alpha} A_{\beta}$. Then $\eta \geq \alpha$. Let $C_{1}$ be the orbit generated by $x_{\eta}$, i.e., $C_{1}=\left\{g^{k}\left(x_{\eta}\right): k \in \mathbb{Z}\right\}$. Since $g\left(\cup_{\beta<\alpha} A_{\beta}\right)=$ $\bigcup_{\beta<\alpha} A_{\beta}$, we have $\bigcup_{\beta<\alpha} A_{\beta}$ and $C_{1}$ disjoint, and according to (13) we can choose $(n-1)$ different orbits $C_{2}, \ldots, C_{n}$ disjoint with $\bigcup_{\beta<\alpha} A_{\beta} \cup C_{1}$. Note 
that all the orbits $C_{1}, \ldots, C_{n}$, as the subset of $A$, are simultaneously either $k_{0}$-cycles (if $k_{0} \neq 0$ ) or infinite (if $k_{0}=0$ ). Put $C=\bigcup_{i=1}^{n} C_{i}$.

Assume first that $\alpha \geq \gamma$ and define $A_{\alpha}=C$. Then (8), (10) and (11) hold, and we construct suitable $\varphi_{\alpha}$ in this case $\alpha \geq \gamma$ as follows. Fix $u_{i}$ in $C_{i}$ for $i=1, \ldots, n$ and define $\varphi_{\alpha}: A_{\alpha} \rightarrow A_{\alpha}$ by putting (in both cases: $k_{0} \neq 0$ and $\left.k_{0}=0\right)$

$$
\varphi_{\alpha}\left(g^{k}\left(u_{i}\right)\right)=g^{k}\left(u_{i+1}\right), \varphi_{\alpha}\left(g^{k}\left(u_{n}\right)\right)=g^{k+1}\left(u_{1}\right)
$$

for $i=1, \ldots, n-1$ and for $k \in \mathbb{Z}$. Clearly, (9) holds.

Consider now the case where $\alpha<\gamma$. According to (5) and (7) we have

$$
\operatorname{card}\left\{y \in A: \operatorname{card} R_{\alpha}^{y}=\operatorname{card} X\right\}=\operatorname{card} X
$$

whereas (13) gives $\operatorname{card}\left(\bigcup_{\beta<\alpha} A_{\beta} \cup C\right)<\operatorname{card} A$. This allows us to fix a $y \in$ $A \backslash\left(\bigcup_{\beta<\alpha} A_{\beta} \cup C\right)$ such that $\operatorname{card} R_{\alpha}^{y}=\operatorname{card} X$. Consequently, denoting $D_{2}=$ $\left\{g^{k}(y): k \in \mathbb{Z}\right\}$, we can find an

$$
x \in R_{\alpha}^{y} \backslash\left(\bigcup_{\beta<\alpha} A_{\beta} \cup C \cup D_{2} \cup A_{-1}\right) .
$$

Put $D_{1}=\left\{g^{k}(x): k \in \mathbb{Z}\right\}$ and choose now $(n-2)$ different orbits $D_{3}, \ldots, D_{n}$ disjoint from $\bigcup_{\beta<\alpha} A_{\beta} \cup C \cup D_{1} \cup D_{2} \cup A_{-1}$. Let $A_{\alpha}=C \cup \bigcup_{i=1}^{n} D_{i}$ and construct now (similarly to the case $\alpha \geq \gamma$ fixing additionally $v_{i} \in D_{i}$ with $\left.v_{1}=x, v_{2}=y\right)$ a function $\varphi_{\alpha}: A_{\alpha} \rightarrow A_{\alpha}$ such that

$$
\varphi_{\alpha}(x)=y
$$

and (9) hold. Then (12) follows from (14) and (15).

According to (11) we have $X=A_{-1} \cup \bigcup_{\alpha<\delta} A_{\alpha}$, and since the summands are disjoint, the formula $\varphi=\varphi_{-1} \cup \bigcup_{\alpha<\delta} \varphi_{\alpha}$ defines a function $\varphi: X \rightarrow X$ which is clearly a solution of (1) and has a big graph with respect to $\mathcal{R}$.

Following [10] consider now a more general equation

$$
\varphi^{n+m}(x)=g\left(\varphi^{m}(x)\right) .
$$

Clearly, every $n$-th iterative root of $g$ is a solution of (16). This simple observation allows us to derive from Theorem 1 the following corollary.

Corollary 1. Assume $m \in \mathbb{N}$. If $\left(\mathrm{H}_{1}\right),\left(\mathrm{H}_{2}\right)$ hold and a family $\mathcal{R}$ of subsets of $X \times X$ satisfies (4) and (5), then there exists a solution $\varphi: X \rightarrow X$ of (16) which has a big graph with respect to $\mathcal{R}$. 
Applying Theorem 1 in the case where $g$ is simply the identity we obtain the following corollary concerning the Babbage equation

$$
\varphi^{n}(x)=x
$$

which belongs to the oldest functional equations (see [11, Ch. XV, §1], [13, 11.7]).

Corollary 2. Assume $X$ is an uncountable set and $\mathcal{R}$ is a family of subsets of $X \times X$ which satisfies (4) and (5). Then for every integer $n \geq 2$ there exists a solution $\varphi: X \rightarrow X$ of the Babbage equation (17) which has a big graph with respect to $\mathcal{R}$.

In the case there $g$ is an involution, i.e., $g^{2}(x)=x$ we have the following corollary which in the very special case of $X=(0,+\infty), g(x)=1 / x$ and $n=2$ generalizes Proposition 5.1 of [5].

Corollary 3. Assume $X$ is uncountable and $g: X \rightarrow X$ is an involution with $L_{1} \neq L_{2} \geq \aleph_{0}$. Let $\mathcal{R}$ be a family of subsets of $X \times X$ which satisfies (4) and (5). Then for every integer $n \geq 2$ there exists a solution $\varphi: X \rightarrow X$ of (1) which has a big graph with respect to $\mathcal{R}$.

The following two remarks give some other conditions which ensure that $\left(\mathrm{H}_{2}\right)$ holds.

Remark 1. Assume that a nonempty set $X$ is equipped with an order $\leq$. If $g: X \rightarrow X$ is a bijection and $g(x)<x$ for every $x \in X$, then $L_{k}=0$ for $k \geq 1$; consequently $\left(\mathrm{H}_{2}\right)$ holds.

Remark 2. Assume that an uncountable set $X$ is equipped with a linear order and $g: X \rightarrow X$ is a bijection.

(i) If $g$ is strictly increasing, then $L_{k}=0$ for $k \geq 2$; if moreover $g$ has less than $\operatorname{card} X$ of fixed points, then $\left(\mathrm{H}_{2}\right)$ holds.

(ii) If $g$ is strictly decreasing, then $L_{k}=0$ for $k \geq 3$; if moreover $n$ is odd and $g^{2}$ has less than card $X$ of fixed points, then $\left(\mathrm{H}_{2}\right)$ holds.

Remark 3. Clearly (cf. also [11, Lemma 15.5] ) any iterative root $\varphi$ of a bijection $g: X \rightarrow X$ maps $k$-cycles onto $k$-cycles and infinite orbits onto infinite orbits and so its graph is a subset of

$$
(X \backslash \operatorname{Per} g)^{2} \cup \bigcup_{k=1}^{\infty} \operatorname{Per}(g, k)^{2} .
$$


Therefore, looking for theorems on the existence of $n$-th iterative roots with big graph in $X \times X$ we have to assume that the set (18) is big in $X \times X$. Our assumption $\left(\mathrm{H}_{2}\right)$ is in this direction.

On the other hand, having a bijection for which $\left(\mathrm{H}_{2}\right)$ does not hold we can ask whether there are solutions having big graph in a subset of $X \times X$ only, e.g., in (18). It turns out that our Theorem 1 jointly with Łojasiewicz's theorem may help in this, as the following corollary shows.

Corollary 4. Assume $\left(\mathrm{H}_{1}\right)$, and for every $k \in \mathbb{N}$, either $L_{k}$ is infinite or divisible by $d_{k}$. Let $\mathcal{R}$ be a family of subsets of (18) such that (4) holds and for every $R \in \mathcal{R}$ we have either

$$
\operatorname{card}\left\{y \in X \backslash \operatorname{Per} g: \operatorname{card} R^{y}=\operatorname{card} X\right\}=\operatorname{card} X
$$

or there exists a $k$ such that

$$
\operatorname{card}\left\{y \in \operatorname{Per}(g, k): \operatorname{card} R^{y}=\operatorname{card} X\right\}=\operatorname{card} X
$$

Then there exists a solution $\varphi: X \rightarrow X$ of (1) which has a big graph with respect to $\mathcal{R}$.

Proof. Put

$\mathcal{R}_{0}=\left\{R \cap(X \backslash \operatorname{Per} g)^{2}: R \in \mathcal{R}, \operatorname{card}\left\{y \in X \backslash \operatorname{Per} g: \operatorname{card} R^{y}=\operatorname{card} X\right\}=\operatorname{card} X\right\}$

and

$\mathcal{R}_{k}=\left\{R \cap \operatorname{Per}(g, k)^{2}: R \in \mathcal{R}, \operatorname{card}\left\{y \in \operatorname{Per}(g, k): \operatorname{card} R^{y}=\operatorname{card} X\right\}=\operatorname{card} X\right\}$

for $k \geq 1$. Fix $k \in \mathbb{N}$. If $\mathcal{R}_{k} \neq \emptyset$ then an application of Theorem 1 to the function $\left.g\right|_{\operatorname{Per}(g, k)}$ (or to $\left.g\right|_{X \backslash \operatorname{Per} g}$ if $k=0$ ) gives a function $\varphi_{k}$ which has a big graph with respect to $\mathcal{R}_{k}$ and such that $\varphi_{k}^{n}=\left.g\right|_{\operatorname{Per}(g, k)}$ (or $\varphi_{k}^{n}=\left.g\right|_{X \backslash \operatorname{Per} g}$ if $k=0$ ). If $\mathcal{R}_{k}=\emptyset$, then using Łojasiewicz's theorem we get a function $\varphi_{k}$ such that $\varphi_{k}^{n}=\left.g\right|_{\operatorname{Per}(g, k)}\left(\right.$ or $\varphi_{k}^{n}=\left.g\right|_{X \backslash \operatorname{Per} g}$ if $\left.k=0\right)$. Putting now $\varphi=\bigcup_{k=0}^{+\infty} \varphi_{k}$ we obtain a solution $\varphi: X \rightarrow X$ of (1) which has a big graph with respect to $\mathcal{R}$.

Example 1. Consider the bijection $g$ on $(-1,1)$ given by $g(x)=x$ for $x \in$ $(-1,0]$ and $g(x)=x^{2}$ for $x \in(0,1)$. According to Corollary 4 there exists a solution $\varphi:(-1,1) \rightarrow(-1,1)$ of $(1)$ which has a big graph with respect to any fixed family $\mathcal{R}$ of subsets of $(-1,0]^{2} \cup(0,1)^{2}$ for which (4) and (5) holds with $X=(-1,1)$. 


\section{Properties of Functions with Big Graph}

Given a topological space $X$, consider the family

$$
\left\{R \in \mathcal{B}(X \times X):\left\{y \in X: R^{y} \text { is uncountable }\right\} \text { is uncountable }\right\}
$$

where $\mathcal{B}(X \times X)$ denotes the $\sigma$-algebra of all Borel subsets of $X \times X$. The following remark is a consequence of the theorem of Alexandrov-Hausdorff ([14, p. 427], [9, 13.6]), the theorem of Mazurkiewicz-Sierpiński ([9, 29.19]) and the fact that there are not more than $2^{\aleph_{0}}$ many Borel sets in a Polish space.

Remark 4. If $X$ is an uncountable Polish space, then the family (19) satisfies all the requirements of Theorem 1 .

The following observation shows that if a function $\varphi: X \rightarrow X$ has a big graph with respect to the family (19), then its graph is big from the topological point of view.

Proposition 1. Assume $\mathrm{T}_{1}$-space $X$ has a countable base and has no isolated point. If $\varphi: X \rightarrow X$ has a big graph with respect to the family (19), then the set $(X \times X) \backslash \operatorname{Gr} \varphi$ contains no subset of $X \times X$ of second category having the property of Baire.

Proof. Assume $(X \times X) \backslash \operatorname{Gr} \varphi$ contains a set $F$ of second category having the property of Baire. Let $G$ be a second category $\mathcal{G}_{\delta}$ subset of $X \times X$ contained in $F$. Consider the sets

$$
\bigcup\left\{G^{y} \times\{y\}: G^{y} \text { is countable }\right\}, \bigcup\left\{G^{y} \times\{y\}: G^{y} \text { is uncountable }\right\}
$$

summing up to $G$. Since $G$ is not in (19), the second one is a countable sum of Borel sets. Consequently both these sets are Borel. Since all the sections of the first one are of first category, the set itself is of first category according to the Kuratowski-Ulam Theorem (see [17, Theorem 15.4], [9, 8.41]). Hence $\left\{x \in X: G_{x}\right.$ is uncountable $\}$ is uncountable, i.e., $G$ belongs to the family (19), a contradiction.

Making use of the Fubini Theorem, instead of that of Kuratowski-Ulam, (and the fact that $\mathcal{B}(X \times X)=\mathcal{B}(X) \times{ }_{\sigma} \mathcal{B}(X)$ if $X$ has a countable base) we obtain the following measure-theoretic analogue of Proposition 1.

Proposition 2. Assume $X$ is the $T_{1}$-space with a countable base. Let $\mu$ and $\nu$ be $\sigma$-finite Borel measures on $X$ vanishing on all the singletons. If $\varphi: X \rightarrow X$ has a big graph with respect to the family (19), then the set $(X \times X) \backslash \operatorname{Gr} \varphi$ contains no Borel subset of $X \times X$ of positive product measure $\mu \times \nu$. 
In other words $(\mu \times \nu)_{*}(X \times X \backslash \operatorname{Gr} \varphi)=0$ and, consequently, $(\mu \times \nu)^{*}(B \cap$ $\operatorname{Gr} \varphi)=(\mu \times \nu)(B)$ for every $B \in \mathcal{B}(X \times X)$. Here $\lambda_{*}$ and $\lambda^{*}$ denote inner and outer measures, respectively, generated by a Borel measure $\lambda$; cf. [7, Sec. 14].

It is worth while to mention that if a Polish space has no isolated point then there are lot of Borel measures on it vanishing on all the singletons $[18$, p. 55].

\section{References}

[1] L. BARTŁOMIEJCZYK, Solutions with big graph of homogeneous functional equations in a single variable, Aequationes Math. 56(1998), 149-156.

[2] L. BARTŁOmiejCZYK, Solutions with big graph of iterative functional equations of the first order, Colloq. Math. 82(1999), 223-230.

[3] L. BArtŁomiejCzyK, Solutions with big graph of an equation of the second iteration, Aequationes Math. (to be published).

[4] L. Barteomiejczyk, Solutions with big graph of the equation of invariant curves, Bull. Polish Acad. Sci. Math. (to be published).

[5] R. Cheng, A. Dasgupta, B. R. Ebanks, L. F. Kinch, L. M. LarSon, And R. B. McFadden, When Does $f^{-1}=1 / f$ ?, Amer. Math. Monthly 105(1998), 704-717.

[6] F. B. Jones, Connected and disconnected plane sets and the functional equation $f(x)+f(y)=f(x+y)$, Bull. Amer. Math. Soc. 48(1942), 115120.

[7] P. R. Halmos, Measure Theory, Graduate Texts in Mathematics 18, Springer-Verlag, New York-Heidelberg-Berlin, 1974.

[8] P. Kahlig And J. Smítal, On the solutions of a functional equation of Dhombres, Results Math. 27(1995), 362-367.

[9] A. S. Kechris, Classical Descriptive Set Theory, Graduate Texts in Mathematics 156, Springer-Verlag, New York-Berlin-Heidelberg, 1994.

[10] M. Kuczma, On some functional equations containing iterations of the unknown function, Ann. Polon. Math. 11(1961), 1-5.

[11] M. Kuczma, Functional Equations in a Single Variable, Monografie Matematyczne 46, PWN-Polish Scientific Publishers, Warszawa, 1968. 
[12] M. Kuczma, An Introduction to the Theory of Functional Equations and Inequalities. Cauchy's Equation and Jensen's Inequality, Prace Naukowe Uniwersytetu Śląskiego w Katowicach 489, Państwowe Wydawnictwo Naukowe \& Uniwersytet Śląski, Warszawa-Kraków-Katowice, 1985.

[13] M. Kuczma, B. Choczewski And R. Ger, Iterative Functional Equations, Encyclopedia of Mathematics and its Applications 32, Cambridge University Press, Cambridge-New York-Port Chester-Melbourne-Sydney, 1990.

[14] K. Kuratowski And A. Mostowski, Set Theory, Studies in Logic and Foundations of Mathematics 86, PWN-Polish Scientific Publishers and North-Holland Publishing Company, Warszawa-Amsterdam-New YorkOxford, 1976.

[15] S. ŁoJASIEwICZ, Solution générale del'équation fonctionelle $f(f(. . f(x) .))=.g(x)$, Ann. Soc. Polon. Math. 24(1951), 88-91.

[16] J. Morawiec, On the existence of irregular solutions of the twocoefficient dilation equation, Aequationes Math. (to be published).

[17] J. C. Oxтовy, Measure and Category, Graduate Text in Mathematics 2, Springer-Verlag, New York-Heidleberg-Berlin, 1971.

[18] K. R. Parthasarathy, Probability Measures on Metric Spaces, Academic Press, New York-San Francisco-London, 1967.

[19] Gy. Targonski, Topics in Iteration Theory, Vandenhoeck \& Ruprecht in Göttingen, 1981. 
